\title{
Modulating reaction pathways on perovskite cobaltite nanofibers through excessive surface oxygen defects for efficient water oxidation
}

\author{
Qiuyu Liu' ${ }^{1}$, Chenghao Jia ${ }^{1}$, Mengzhen Zhou ${ }^{1}$, Zuyun He ${ }^{1}$, Zhiheng Gong ${ }^{1}$, Yihan \\ Ling ${ }^{2}$, Xinxin Wang $^{2}$, Keiji Yashiro ${ }^{3}$, Yan Chen ${ }^{* 1}$
}

${ }^{1}$ School of Environment and Energy, State Key Laboratory of Pulp and Paper

Engineering, South China University of Technology, Guangzhou, 510006, China

${ }^{2}$ School of Materials Science and Physics, China University of Mining and

Technology, Xuzhou, 221116, PR China

${ }^{3}$ Graduate School of Environmental Studies, Tohoku University, Sendai, 980-8577, Japan

\section{Experimental Section}

SNCF powders synthesized through solid-state method

SNCF powders reference sample were synthesized through a traditional solid-state method. Stoichiometric amounts of $\mathrm{SrCO}_{3}, \mathrm{Nb}_{2} \mathrm{O}_{5}, \mathrm{Co}_{2} \mathrm{O}_{3}$ and $\mathrm{Fe}_{2} \mathrm{O}_{3}$ were mixed with ethanol and milled for $12 \mathrm{~h}$. The obtained mixture was dried and then calcined at $1200{ }^{\circ} \mathrm{C}$ in air for $10 \mathrm{~h}$. After calcination, the powders was refined by further ballmilling for $24 \mathrm{~h}$.

\section{SNCF nanofibers synthesized by eletrospinning}

SNCF nanofibers were prepared by using electrospinning method. Stoichiometric amounts of $\mathrm{Sr}\left(\mathrm{NO}_{3}\right)_{2}, \mathrm{C}_{4} \mathrm{H}_{4} \mathrm{NNbO}_{9} \cdot \mathrm{xH}_{2} \mathrm{O}, \mathrm{Fe}\left(\mathrm{NO}_{3}\right)_{3} \cdot 9 \mathrm{H}_{2} \mathrm{O}$ and $\mathrm{Co}\left(\mathrm{NO}_{3}\right)_{2} \cdot 6 \mathrm{H}_{2} \mathrm{O}$ were dissolved in DMF as precursor solution. Then, PVP was added to the solution which 
were polarized to be charged and drawn in an electric field during the electrospinning process. The mixture was stirred overnight to ensure dissolution of precursor materials. The as-prepared SNCF precursor solution was loaded into a plastic syringe equipped with the stainless steel needle of different sizes to control the diameter of the nanofibers. The applied voltage was fixed to $17.0 \mathrm{kV}$, and the electrospinning fibers were collected on a piece of aluminum foil. The rotation speed of drum was set to be constant at $150 \mathrm{rpm}$. The receiving distance between was $20 \mathrm{~cm}$. After electrospining, the precursor materials were dried and then calcined at $800{ }^{\circ} \mathrm{C}$ for $2 \mathrm{~h}$ at a heating rate of $2{ }^{\circ} \mathrm{C} \mathrm{min}^{-1}$. The detailed experimental parameters are shown in Table S1

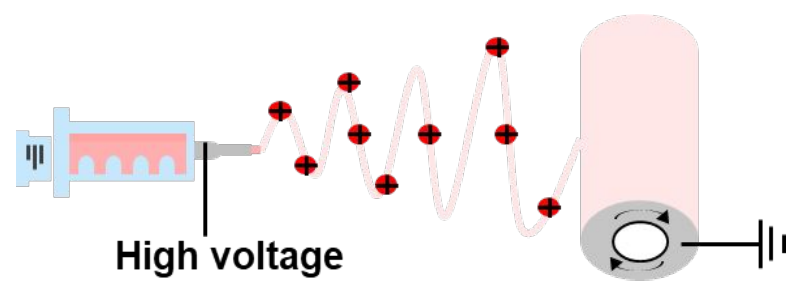

Figure S1. Principle of electrospinning
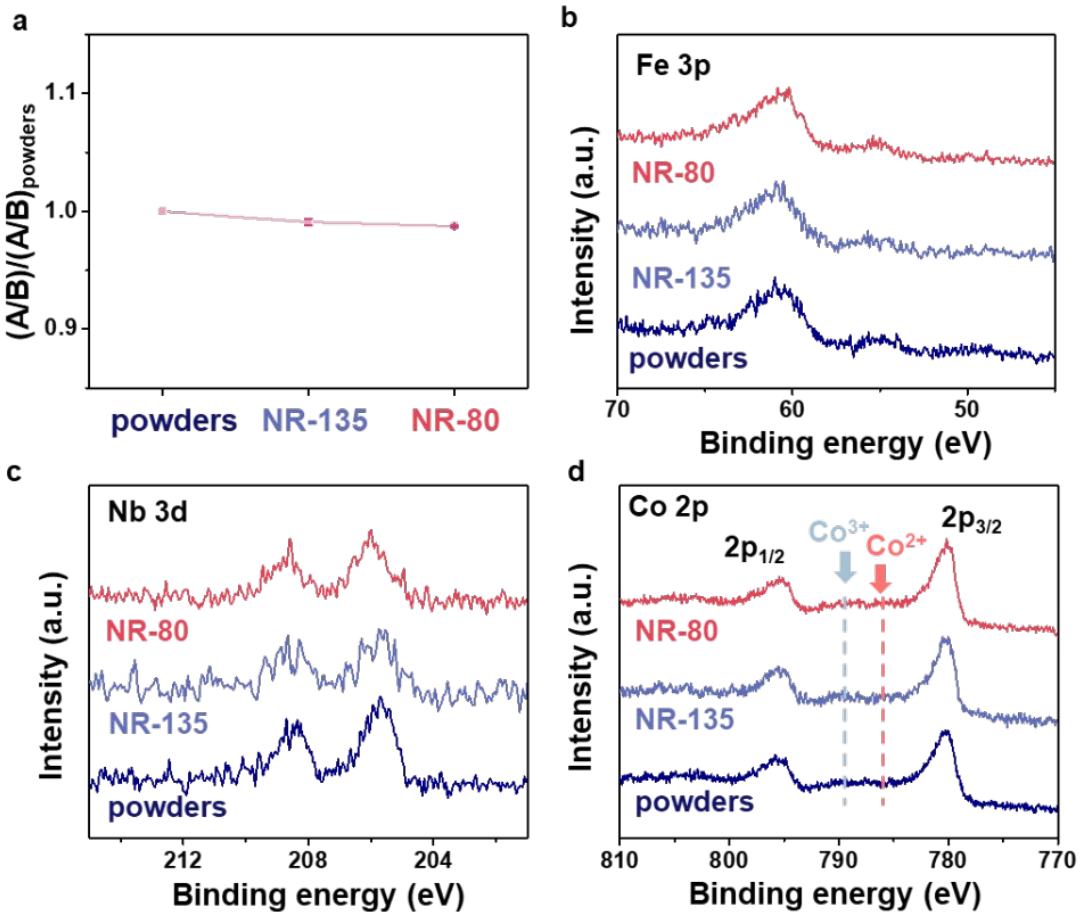

Figure S2. (a) The radio of (A/B)/(A/B) powders for powders, NR-135 and NR-80. (b-d), $\mathrm{Fe} 3 \mathrm{p}, \mathrm{Nb} 3 \mathrm{~d}$ and $\mathrm{Co} 2 \mathrm{p}$ XPS spectra for powders, NR-135 and NR-80; 

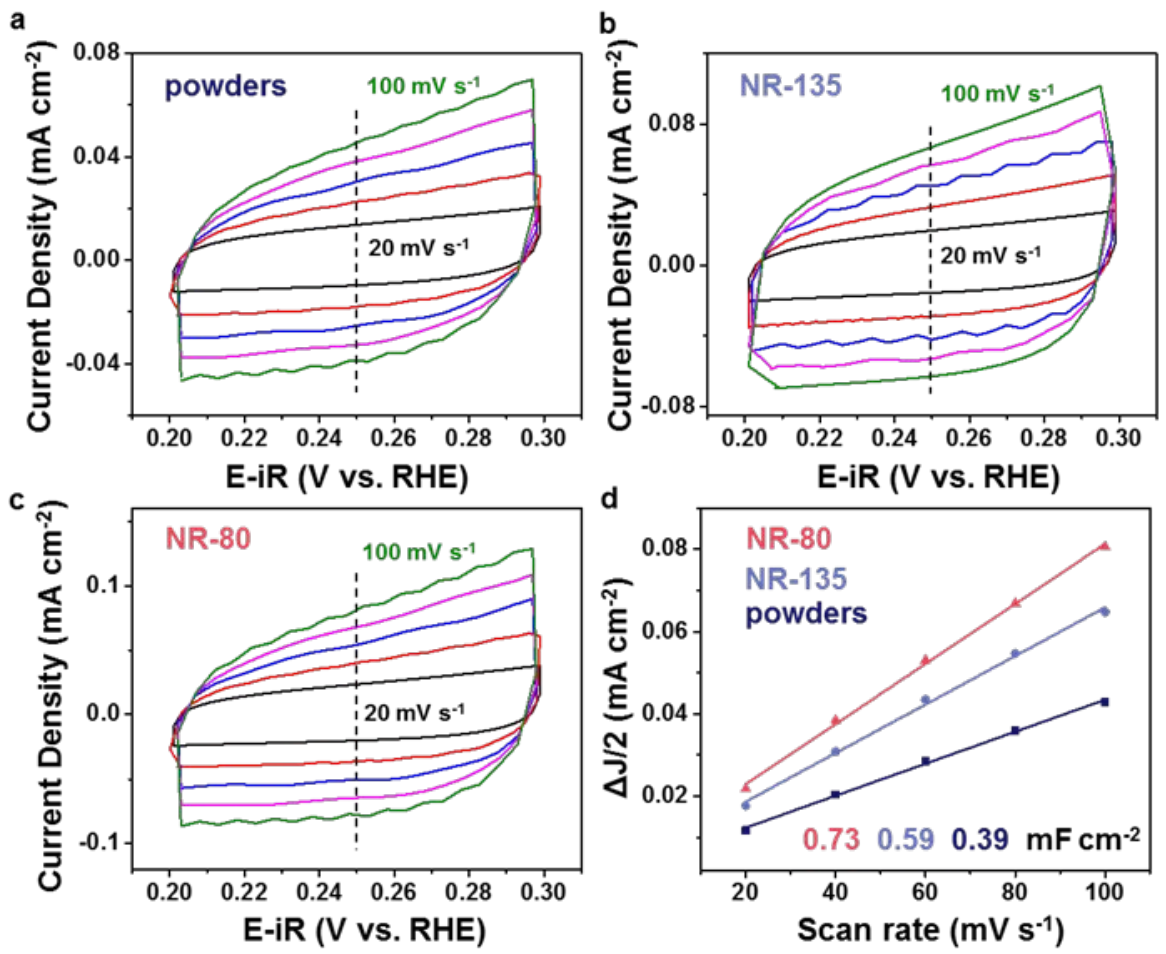

Figure. S3 CV measurements in a non-faradic current region for (a) powders, (b) NR135 and (c) NR-80. (d) $C_{\text {dl }}$ plots of powders, NR-135 and NR-80. To calculate the electrochemical double-layer capacitances $\left(\mathrm{C}_{\mathrm{dl}}\right)$, cyclic voltammograms $(\mathrm{CVs})$ were recorded in the potential window of 0.2 to $0.3 \mathrm{~V}$ ( $\mathrm{vs}$. $\mathrm{Ag} / \mathrm{AgCl}$ ) with scan rates ranging from 20 to $100 \mathrm{mV} \mathrm{s}^{-1}$. ECSA were calculated based on ECSA $=\mathrm{C}_{\mathrm{dl}} / \mathrm{C}_{\mathrm{s}}$, where $\mathrm{C}_{\mathrm{dl}}$ is double-layer capacitance, $C_{s}$ is a general specific capacitance with the value of $0.04 \mathrm{mF}$ $\mathrm{cm}^{-2} \cdot{ }^{1-2}$

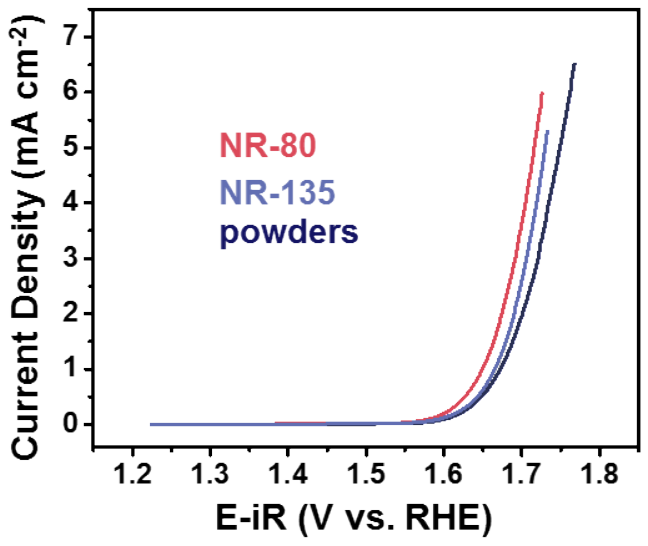

Figure S4. LSV curves (current density normalized by ECSA) of powders, NR-135 
and NR-80.
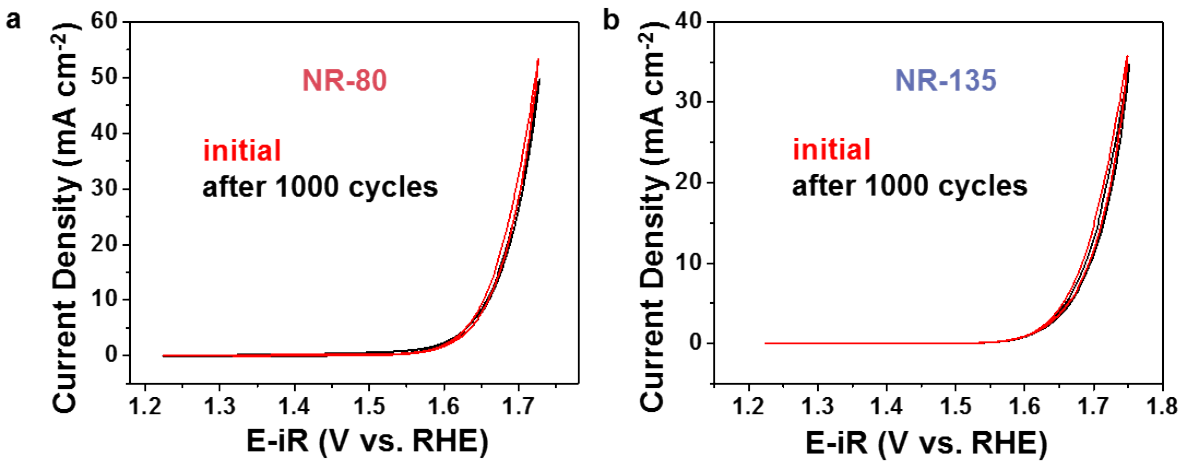

Figure S5. CVs of NR-80 and NR-135 catalysts initially and after 1000 cycles.

Table S1. Electrospinning Parameters of preparing SNCF nanofibers

\begin{tabular}{cccccc}
\hline Simple & n/nmol & PVP/g & DMF/mL & Needle & Feeding rate/uL min $^{-1}$ \\
\hline NR-135 & 5 & 5 & 32.5 & $24 \mathrm{G}$ & 0.30 \\
NR-80 & 5 & 5 & 37.5 & $21 \mathrm{G}$ & 0.15 \\
\hline
\end{tabular}

Table S2. O 1s XPS peak deconvolution results.

\begin{tabular}{ccccc}
\hline Sample & Lattice $\mathrm{O}$ & $\mathrm{O}$ defect & Surface $\mathrm{OH}^{-} / \mathrm{O}_{2}$ & Surface $\mathrm{H}_{2} \mathrm{O}$ \\
\hline NR-80 & $6.27 \%$ & $20.52 \%$ & $66.68 \%$ & $6.53 \%$ \\
NR-135 & $10.11 \%$ & $17.18 \%$ & $57.40 \%$ & $15.31 \%$ \\
powders & $22.80 \%$ & $11.85 \%$ & $56.42 \%$ & $8.93 \%$ \\
\hline
\end{tabular}

Table S3. Electrochemical impedance spectroscopy (EIS) fitting results for NR-80, NR-135 and powders.

\begin{tabular}{cccc}
\hline Sample & $\mathrm{R}_{\mathrm{s}}(\Omega)$ & $\mathrm{R}_{\mathrm{ct}}(\Omega)$ & $\mathrm{CPE}$ \\
\hline NR-80 & 1.0 & 2.5 & 0.78 \\
NR-135 & 1.0 & 3.6 & 0.76 \\
Powders & 1.1 & 7.3 & 0.78 \\
\hline
\end{tabular}


Table S4. Comparison of the OER activity for different catalysts. The turnover frequency (TOF) for NR-80 and NR-135 was calculated by TOF $=j A / 4 F n$, where $j$ is the OER current at $1.68 \mathrm{~V}$ vs. RHE, $A$ is the geometric area of the working electrode, $F$ is the Faraday constant, and $n$ is the total number of moles of cobalt and iron atoms on the working electrode. ${ }^{3-5}$

\begin{tabular}{|c|c|c|c|c|}
\hline Material & $\begin{array}{l}\text { Overpotential } \\
\eta @ 10 \mathrm{~mA} \\
\mathrm{~cm}^{-2}(\mathrm{~V})\end{array}$ & $\begin{array}{c}\text { Tafel } \\
\text { slope (mV } \\
\left.\operatorname{dec}^{-1}\right)\end{array}$ & TOF $\left(\mathrm{s}^{-1}\right)$ & Ref \\
\hline NR-80 & 0.423 & 43.6 & $\begin{array}{c}0.0606 \text { at } \eta= \\
450 \mathrm{mV}\end{array}$ & This work \\
\hline NR-135 & 0.447 & 49.3 & $\begin{array}{c}0.0318 \text { at } \eta= \\
450 \mathrm{mV}\end{array}$ & This work \\
\hline $\mathrm{LaFeO}_{3}$ & 0.470 & 72 & $\begin{array}{c}0.0200 \text { at } \eta= \\
450 \mathrm{mV}\end{array}$ & 6 \\
\hline $\mathrm{LaFe}_{0.8} \mathrm{Co}_{0.2} \mathrm{O}_{3}$ & 0.460 & 70 & $\begin{array}{c}0.0240 \text { at } \eta= \\
450 \mathrm{mV}\end{array}$ & 6 \\
\hline $3 \mathrm{DOM}^{-\mathrm{LaFeO}_{3}}$ & 0.420 & 62 & $\begin{array}{c}0.0450 \text { at } \eta= \\
450 \mathrm{mV}\end{array}$ & 6 \\
\hline 3DOM- & 0.410 & 56 & 0.0620 at $\eta=$ & 6 \\
\hline $\mathrm{LaFe}_{0.8} \mathrm{Co}_{0.2} \mathrm{O}_{3}$ & & & $450 \mathrm{mV}$ & \\
\hline NiFe-LDH & 0.350 & 40 & $\begin{array}{c}0.0500 \text { at } \eta= \\
300 \mathrm{mV}\end{array}$ & 7 \\
\hline FeP@Au & 0.320 & 56.8 & $\begin{array}{c}0.0040 \text { at } \eta= \\
330 \mathrm{mV}\end{array}$ & 8 \\
\hline $\mathrm{IrO}_{2}$ & 0.340 & 46 & $\begin{array}{c}0.0110 \text { at } \eta= \\
300 \mathrm{mV}\end{array}$ & 7 \\
\hline
\end{tabular}




\section{REFERENCES}

(1) Zhu, Y.; Tahini, H. A.; Hu, Z.; Dai, J.; Chen, Y.; Sun, H.; Zhou, W.; Liu, M.; Smith, S. C.; Wang, H.; Shao, Z., Unusual synergistic effect in layered RuddlesdenPopper oxide enables ultrafast hydrogen evolution. Nat. Commun. 2019, 10 (1), 149.

(2) Nadeema, A.; Walko, P. S.; Devi, R. N.; Kurungot, S., Alkaline Water Electrolysis by NiZn-Double Hydroxide-Derived Porous Nickel Selenide-Nitrogen-Doped Graphene Composite. ACS Appl. Energy Mater. 2018.

(3) Dai, J.; Zhu, Y.; Zhong, Y.; Miao, J.; Lin, B.; Zhou, W.; Shao, Z., Enabling High and Stable Electrocatalytic Activity of Iron-Based Perovskite Oxides for Water Splitting by Combined Bulk Doping and Morphology Designing. Adv. Mater. Interfaces 2019, 6 (1), 1801317.

(4) Zhang, J.; Hu, Y.; Liu, D.; Yu, Y.; Zhang, B., Enhancing Oxygen Evolution Reaction at High Current Densities on Amorphous-Like Ni-Fe-S Ultrathin Nanosheets via Oxygen Incorporation and Electrochemical Tuning. Adv. Sci. 2017, 4 (3), 1600343.

(5) Hirai, S.; Yagi, S.; Chen, W. T.; Chou, F. C.; Okazaki, N.; Ohno, T.; Suzuki, H.; Matsuda, T., Non-Fermi Liquids as Highly Active Oxygen Evolution Reaction Catalysts. Adv. Sci. 2017, 4 (10), 1700176.

(6) Dai, J.; Zhu, Y.; Zhong, Y.; Miao, J.; Lin, B.; Zhou, W.; Shao, Z., Enabling High and Stable Electrocatalytic Activity of Iron - Based Perovskite Oxides for Water Splitting by Combined Bulk Doping and Morphology Designing. Adv. Mater. Interfaces 2018, 6 (1).

(7) Song, F.; Hu, X., Exfoliation of layered double hydroxides for enhanced oxygen evolution catalysis. Nat. Commun. 2014, 5, 4477.

(8) Masud, J.; Umapathi, S.; Ashokaan, N.; Nath, M., Iron phosphide nanoparticles as an efficient electrocatalyst for the OER in alkaline solution. J. Mater. Chem. A 2016, 4 (25), 9750-9754. 